\title{
NONLINEAR HYPERBOLIC EQUATIONS IN INFINITE HOMOGENEOUS WAVEGUIDES
}

\author{
JASON METCALFE, CHRISTOPHER D. SOGGE, AND ANN STEWART
}

\begin{abstract}
In this paper we prove global and almost global existence theorems for nonlinear wave equations with quadratic nonlinearities in infinite homogeneous waveguides. We can handle both the case of Dirichlet boundary conditions and Neumann boundary conditions. In the case of Neumann boundary conditions we need to assume a natural nonlinear Neumann condition on the quasilinear terms. The results that we obtain are sharp in terms of the assumptions on the dimensions for the global existence results and in terms of the lifespan for the almost global results. For nonlinear wave equations, in the case where the infinite part of the waveguide has spatial dimension three, the hypotheses in the theorem concern whether or not the Laplacian for the compact base of the waveguide has a zero mode or not.
\end{abstract}

\section{Introduction.}

In this paper we shall consider nonlinear wave and Klein-Gordon equations of the form

$$
\left\{\begin{array}{l}
\left(\square+m^{2}\right) u=Q\left(u, u^{\prime}, u^{\prime \prime}\right), \\
u(0, x)=f(x), \quad \partial_{t} u(0, x)=g(x) .
\end{array}\right.
$$

where

$$
\square=\partial_{t}^{2}-\left(\Delta+\Delta_{\Omega}\right)
$$

is the d'Alembertian on $(t, x, y) \in \mathbb{R}_{+} \times \mathbb{R}^{n} \times \Omega$. Here,

$$
\Delta=\Delta_{\mathbb{R}^{n}}=\sum_{j=1}^{n} \partial^{2} / \partial x_{j}^{2}
$$

is the Euclidean Laplacian on $\mathbb{R}^{n}$. Also, $\Omega \subset \mathbb{R}^{d}$ denotes a nonempty, bounded domain with smooth boundary $\partial \Omega$, and $\Delta_{\Omega}$ denotes the standard Laplacian

$$
\Delta_{\Omega}=\sum_{j=1}^{d} \partial^{2} / \partial y_{j}^{2} .
$$

So we shall impose either Dirichlet boundary conditions

$$
\left.u(t, x, y)\right|_{y \in \partial \Omega}=0
$$

or Neumann boundary conditions

$$
\left.\partial_{\nu} u(t, x, y)\right|_{y \in \partial \Omega}=0
$$

The authors were supported in part by the NSF. 
with $\partial_{\nu}$ denoting the normal derivative at $\partial \Omega$. Thus, depending on which case we are in, $\Delta_{\Omega}$ will be either the Dirichlet Laplacian or the Neumann Laplacian on $\Omega$.

Also, in (1.1), $Q$ is a quadratic function in its arguments, which are $u, u^{\prime}=\nabla u$, and $u^{\prime \prime}=\nabla^{2} u$, with $\nabla=\left(\partial_{t}, \partial_{x}, \partial_{y}\right)$ being the full space-time gradient. We shall assume that our nonlinear equations are quasilinear, which means that they are affine linear in $u^{\prime \prime}$. For simplicity we shall just state things for the scalar case, but everything carries over without any difficult to the case where (1.1) is a nonlinear system in $u=\left(u^{1}, \ldots, u^{N}\right)$. So, we are assuming that $Q$ can be written as

$$
Q\left(u, u^{\prime}, u^{\prime \prime}\right)=\sum_{0 \leq j, k, l \leq n+d} A_{l}^{j k} \partial_{l} u \partial_{j} \partial_{k} u+u \sum_{0 \leq j, k \leq n+d} A^{j k} \partial_{j} \partial_{k} u+R\left(u, u^{\prime}\right)
$$

where $R$ is a quadratic function in $u$ and $u^{\prime}$. Here, and in what follows, $x_{0}=t$, and $x_{n+j}=y_{j}, 1 \leq j \leq d$.

In order to solve (1.1) we must assume that the data satisfies the relevant compatibility conditions. Since these are well known (see, e.g., [7]), we shall describe them briefly. To do so we first let $J_{k} u=\left\{\partial_{x}^{\alpha} u: 0 \leq|\alpha| \leq k\right\}$ denote the collection of all spatial derivatives of $u$ of order up to $k$, using local coordinates in a small tubular neighborhood of $\partial \Omega$. Then if $N$ is fixed and if $u$ is a formal $H^{N}$ solution of 1.1 we can write $\partial_{t}^{k} u(0, \cdot)=$ $\psi_{k}\left(J_{k} f, J_{k-1} g\right), 0 \leq k \leq N$, for certain compatibility functions $\psi_{k}$ which depend on the nonlinear term $Q$ as well as $J_{k} f$ and $J_{k-1} g$. Having done this, the compatibility condition for the Dirchlet case (1.1), (1.3) with $(f, g) \in H^{N} \times H^{N-1}$ is just the requirement that the $\psi_{k}$ vanish on $\mathbb{R}_{+} \times \mathbb{R}^{n} \times \partial \Omega$ when $0 \leq k \leq N-1$. Additionally, we shall say that $(f, g) \in C^{\infty}$ satisfy the compatibility conditions to infinite order if this condition holds for all $N$. For the Neumann case (1.1), (1.4) one follows a similar construction by writing $\partial_{t}^{k} \partial_{\nu} u(0, \cdot)=\Psi_{k}\left(J_{k+1} f, J_{k} g\right)$ and requiring that the $\Psi_{k}$ vanish on $\mathbb{R}_{+} \times \mathbb{R}^{n} \times \partial \Omega$ when $0 \leq k \leq N-2$.

For simplicity, we shall also assume that the initial data has compact support. So we shall assume that there is a fixed constant $B>0$ so that

$$
f(x, y)=g(x, y)=0, \quad|x|>B,
$$

where we recall that $x$ denotes the $\mathbb{R}^{n}$ variable. In addition to (1.6) we shall also have to assume that the initial data is small,

$$
\|f\|_{H^{N}\left(\mathbb{R}^{n} \times \Omega\right)}+\|g\|_{H^{N-1}\left(\mathbb{R}^{n} \times \Omega\right)} \leq \varepsilon
$$

where $\|f\|_{H^{N}}=\sum_{|\alpha| \leq N}\left\|\partial_{x, y}^{\alpha} f\right\|_{L^{2}\left(\mathbb{R}^{n} \times \Omega\right)}$. This equation can be relaxed in certain situations to a condition that certain weighted-Sobolev norms are small.

Let us now state our main results. The first says that for Dirichlet boundary conditions we always have small amplitude global existence for Klein-Gordon or wave equations with quadratic nonlinearities when $n \geq 3$.

Theorem 1.1. Suppose that $m \geq 0$ in (1.1), and assume that $n \geq 3$. Assume also that the Cauchy data $(f, g) \in C^{\infty}\left(\mathbb{R}^{n} \times \Omega\right)$ satisfies (1.6) and (1.7) as well as the appropriate infinite order compatibility conditions for the Dirichlet boundary conditions (1.3). It then follows that the corresponding nonlinear hyperbolic equation (1.1), (1.3) has a global smooth solution if $N$ in (1.7) is a sufficiently large fixed integer and if $0<\varepsilon<\varepsilon_{0}$ is sufficiently small. 
The case of Neumann boundary conditions is more delicate for several reasons. First, to be able to have energy estimates, one needs a natural nonlinear Neumann condition on the quasilinear quadratic forms (see $§ 5$ )

$$
\sum_{0 \leq j, k, l \leq n+d} A_{l}^{j k} \xi_{l} \eta_{j} \theta_{k}=0 \quad \text { and } \sum_{0 \leq j, k \leq n+d} A^{j k} \xi_{j} \theta_{k}=0, \quad \text { if }(\theta, \xi, \eta) \in X,
$$

where

$$
X=\left\{(\theta, \xi, \eta): \theta=\left(0, \ldots, 0, \nu_{1}(y), \ldots, \nu_{d}(y)\right), \xi \cdot \theta=0, \eta \cdot \theta=0, y \in \partial \Omega\right\} .
$$

Thus, $\theta$ is assumed to be normal to $\mathbb{R}^{1+n} \times \partial \Omega$, and $\xi$ and $\eta$ are both assumed to be orthogonal to $\theta$. Note that this condition automatically holds if the quasilinear terms only involve $\partial_{j} \partial_{k} u, 0 \leq j, k \leq n$, i.e., $A_{l}^{j k}=0$ and $A^{j k}=0$ if $n+1 \leq j \leq n+d$ or $n+1 \leq k \leq n+d$. This condition is the natural one so that energy estimates can hold. It should be compared to the symmetry condition for multispeed nonlinear hyperbolic systems (see, e.g., [9], [16], [20]).

If we assume this nonlinear condition, then in the case of Neumann boundary conditions we get the same sort of results for Klein-Gordon (i.e., $m>0$ ) equations:

Theorem 1.2. Suppose that $m>0$ in (1.1), and assume that $n \geq 3$. Assume also that the Cauchy data $(f, g) \in C^{\infty}\left(\mathbb{R}^{n} \times \Omega\right)$ satisfies (1.6) and (1.7) as well as the appropriate infinite order compatibility conditions for the Neumann boundary conditions (1.4). Then, if the nonlinear Neumann condition (1.8) is satisfied, it follows that the corresponding nonlinear Klein-Gordon equation (1.1), (1.4) has a global smooth solution if $N$ in (1.7) is a sufficiently large fixed integer and if $0<\varepsilon<\varepsilon_{0}$ is sufficiently small.

Proving existence theorems for nonlinear wave equations (i.e., $m=0$ ) in waveguides with Neumann boundary conditions is more difficult. However, we can obtain optimal results for certain semilinear equations without too much difficulty using ideas from [8].

Theorem 1.3. Consider the nonlinear wave equation (1.1) with $m=0$. Assume that the quadratic nonlinearity is independent of $u, \partial_{t} u, \nabla_{y} u$ and $u^{\prime \prime}$, i.e., that (1.1) is replaced by

$$
\square u=Q\left(\nabla_{x} u\right),
$$

where $Q\left(\nabla_{x} u\right)$ is a bilinear form in the spatial $\mathbb{R}^{n}$ gradient. Assume that the data $(f, g)$ are as in Theorem 1.2. Then if $n \geq 4$ (1.9), (1.4) has a global smooth solution if $\varepsilon<\varepsilon_{0}$ is sufficiently small. If $n=3$ and $\varepsilon<\varepsilon_{0}$ is sufficiently small, there is a constant $c>0$ so that if

$$
T_{\varepsilon}=\exp (c / \varepsilon),
$$

then (1.9), (1.4) has a solution $u \in C^{\infty}\left(\left[0, T_{\varepsilon}\right) \times \mathbb{R}^{3} \times \Omega\right)$.

This result is of course optimal. If one takes data $f(x, y)=f(x), g(x, y)=g(x)$ that are independent of $y \in \Omega$ then the solution of the equation (1.9), (1.4) is given by $u(t, x, y)=u(t, x)$ where $u$ is the solution of the equation $\square u(t, x)=Q\left(\nabla_{x} u(t, x)\right)$ in Minkowski space. Classic results of John (see, e.g., [5, 6]) show that there is blowup for times of order $\exp (C / \varepsilon)$ when $n=3$ and $Q\left(\nabla_{x} u\right)=\left|\nabla_{x} u\right|^{2}$.

It is for technical reasons that we can only handle semilinear terms involving $\nabla_{x} u$ in dimensions $n=3,4$. We remark, though, that when $n \geq 5$ it seems that our extension 
(2.5) of the KSS inequality 8 does give global existence for equations involving bilinear forms in $\left(\partial_{t} u, \nabla_{x} u\right)$. We shall study this in a future paper.

We remark that we could also have obtained the analog of Theorem 1.2 or Theorem 1.3 in the case where $\Omega$ is a compact Riemannian manifold without boundary. As we shall see in the proof the case of Dirichlet boundary conditions is much more favorable than the boundaryless case or the case of Neumann boundary conditions when $m=0$. This is because the latter cases have zero eigenmodes. In the case of Dirichlet boundary conditions, since there are no zero eigenvalues, one can naturally reduce matters to proving uniform estimates for Klein-Gordon equations $\square_{\mathbb{R}^{1+n}}+\mu^{2}$ in $\mathbb{R} \times \mathbb{R}^{n}$ with $\mu \geq 1$. On the other hand, if $m=0$, then for the Neumann or boundaryless case, one must also consider estimates for $\square_{\mathbb{R}^{1+n}}$, which are less favorable in terms of time-decay. For example, see the Klainerman-Sobolev inequalities [10] (see also, e.g., 2] and 22]) which roughly provide $t^{-(n-1) / 2}$ decay for the wave equation as compared to the $t^{-n / 2}$ decay in Proposition 2.1 and Proposition [2.2 for the Klein-Gordon equation. Here, and throughout, $\square_{\mathbb{R}^{1+n}}=\partial_{t}^{2}-\Delta_{\mathbb{R}^{n}}$.

In order to show global existence of solutions to the nonlinear problem (1.1) in the proofs of Theorem 1.1 and Theorem 1.2 we will use the above a priori estimates and an energy estimate in a fashion similar to that used by Klainerman [1] in the boundaryless case. See also Hörmander 2. It should be noted that Shatah 18 provides an alternate proof in the boundaryless case that uses normal forms to reduce to the case of nonlinearities vanishing to third order. From here, the proof proceeds using estimates that are relatively straightforward as compared to the a priori estimates used in [1]. We, however, do not explore Shatah's method in this paper. As mentioned previously, the proof of global existence for wave equations in waveguides with Neumann boundary conditions is more difficult. A proof of Theorem 1.3 which uses techniques reminiscent of those of [8] is provided.

Earlier results were obtained by Lesky and Racke 13, and their work inspired this one. Their techniques largely relied on $L^{p} \rightarrow L^{p /(p-1)}$ decay estimates for the linear KleinGordon equation that are reminiscent to ones obtained earlier by Marshall, Strauss and Wainger 14. As with our estimates, the main point is to prove estimates for solutions of Klein-Gordon equations $\left(\square_{\mathbb{R}^{1+n}}+m^{2}\right) u=0$ in $\mathbb{R} \times \mathbb{R}^{n}$ that are uniform in $m \geq 1$. By using an eigenfunction expansion for the other part of the waveguide, $\Omega$, one can use these to easily obtain estimates on $\mathbb{R} \times\left(\mathbb{R}^{n} \times \Omega\right)$. Then, using techniques of Shibata and Tsutsumi [19] they are able to use these linear estimates to show that the above global existence results hold in the case of Dirichlet boundary conditions when $n \geq 5$. If $n$ is fixed then as the dimension of $\Omega$ increases, one of course needs more and more regularity of the initial data.

Our techniques are related to recent work on nonlinear obstacle problems, (e.g., 7], 8, 9], 16, and 21]). However, the results here are considerably easier to obtain for a couple of reasons. First, and most important, when we use the method of commuting vector fields (see, e.g., 22]) in our case, since there is no obstacle in $\mathbb{R}^{n}$, we are allowed to use the generators of hyperbolic rotations, i.e.,

$$
\Omega_{0 j}=x_{j} \partial_{t}+t \partial_{j}, \quad 1 \leq j \leq n
$$


as well as the generators of spatial rotations,

$$
\Omega_{j k}=x_{j} \partial_{k}-x_{k} \partial_{j}, \quad 1 \leq j<k \leq n .
$$

Also since there is no obstacle in $\mathbb{R}^{n}$, we do not have to use the unit cutoff method that relies on local exponential decay of energy ([3, 4], 12, 17]). If one wishes to extend our results to the case of inhomogeneous wave guides where one considers a compact perturbation of the $\mathbb{R}^{n}$-Laplacian, either from a change of metric or an obstacle, then the situation would be much more delicate. We should also point out that in our situation we cannot use the scaling vector field $t \partial_{t}+r \partial_{r}$ since it does not preserve the equation $\left(\partial_{t}^{2}-\Delta+\mu^{2}\right) u=0, \mu \neq 0$. However, this presents no problems since the Sobolev estimates and weighted $L^{2}(d t d x)$ estimates are strong enough. The latter follow easily from a slight variant of a local estimate of Smith and the second author 21.

The paper is organized as follows. In the next two sections, we shall state the estimates for the linearized equation that we shall need. We first prove estimates in $\mathbb{R}^{n}$, including ones which involve bounds that are uniform in the mass parameter $m$ for the linear equation. We then show how these uniform bounds easily lead to estimates for solutions of linear equations in waveguides. Using these uniform estimates and orthogonality we can easily obtain the existence theorems using standard arguments. These proofs are carried out in $\S 4$ for the Dirichlet case and in $\S 5$ for the Neumann cases. In what follows, $C$ shall denote a constant which can change at each occurrence.

\section{Linear Estimates in $\mathbb{R}_{+} \times \mathbb{R}^{n}$.}

To handle the existence results for Klein-Gordon equations or Dirichlet-wave equations, we shall need estimates which follow immediately from estimates in Hörmander [2] To be more precise, if

$$
\left\{\Gamma_{t, x}\right\}=\left\{\partial_{t}, \partial_{x}, \Omega_{j k}: 0 \leq j<k \leq n\right\}
$$

then we need the following estimate to handle the case where $n=3$.

Proposition 2.1. Suppose that $u \in C^{\infty}\left(\mathbb{R} \times \mathbb{R}^{3}\right)$ satisfies $u(t, x)=0, t \leq 2 B$, where $B$ is a fixed positive constant. Suppose also that $\left(\square_{\mathbb{R}^{1+3}}+\mu^{2}\right) u(t, x)=0$ for $|x|>t-B$, Then there is a constant depending only on $B$ so that when $\mu \geq 1$

$$
\sup _{x} t^{3 / 2}|u(t, x)| \leq C \sum_{|\alpha| \leq 5} \sum_{k} \sup _{\tau \in\left[2^{k-1}, 2^{k+1}\right] \cap[2 B, t]} 2^{k}\left\|\Gamma_{t, x}^{\alpha}\left(\square_{\mathbb{R}^{1+3}}+\mu^{2}\right) u(\tau, \cdot)\right\|_{2}
$$

This follows exactly from the proof of Proposition 7.3.6 in Hörmander 2 if one uses a variation of Lemma 7.3.4 there. The relevant version for (2.2) is that if $w^{\prime \prime}+\mu^{2} w=h$ in $[a, b] \subset \mathbb{R}$, then

$$
\sup _{a \leq \rho \leq b}|w(\rho)| \leq|w(a)|+\left|w^{\prime}(a)\right|+\frac{1}{\mu} \int_{a}^{b}|h(\rho)| d \rho .
$$

In a similar manner one obtains the following analog of Proposition 7.3.7 in [2] for the case where $n \geq 4$.

Proposition 2.2. Suppose that $n \geq 4$ and that $u \in C^{\infty}\left(\mathbb{R} \times \mathbb{R}^{n}\right)$ satisfies $u(t, x)=0$, $t \leq 2 B$, where $B$ is a fixed positive constant. Suppose also that $\left(\square_{\mathbb{R}^{1+n}}+\mu^{2}\right) u(t, x)=0$ 
for $|x|>t-B$. Then if $n=4$ there is a constant depending only on $B$ so that when $\mu \geq 1$

$$
\sup _{x} t^{2}|u(t, x)| \leq C \sum_{|\alpha| \leq 7} \sum_{k} \sup _{\tau \in\left[2^{k-1}, 2^{k+1}\right] \cap[2 B, t]}(1+|k|) 2^{k}\left\|\Gamma_{t, x}^{\alpha}\left(\square_{\mathbb{R}^{1+4}}+\mu^{2}\right) u(\tau, \cdot)\right\|_{2}
$$

If $n \geq 5$ there is a constant depending only on $B$ and $n$ so that when $\mu \geq 1$

$$
\sup _{x} t^{1+\frac{n}{4}}|u(t, x)| \leq C \sum_{|\alpha| \leq n+3} \sum_{k} \sup _{\tau \in\left[2^{k-1}, 2^{k+1}\right] \cap[2 B, t]} 2^{k}\left\|\Gamma_{t, x}^{\alpha}\left(\square_{\mathbb{R}^{1+n}}+\mu^{2}\right) u(\tau, \cdot)\right\|_{2}
$$

To prove Theorem 1.3 (i.e., Neumann boundary conditions with $m=0$ ), we shall also require weighted $L_{t}^{2} L_{x}^{2}$ estimates of the type that were first used in [8]. Here and throughout, we will use the notation $\langle x\rangle=\langle r\rangle=\sqrt{1+|x|^{2}}$.

Proposition 2.3. Fix $n \geq 3$. Then there is a uniform constant $C$ which is independent of $\mu \geq 0$ so that if $u \in C^{\infty}\left(\mathbb{R}_{+} \times \mathbb{R}^{n}\right)$ vanishes for $t \leq 0$ and vanishes for large $x$ for every fixed then

$$
\begin{aligned}
&\left(\int _ { 0 } ^ { T } \int _ { \mathbb { R } ^ { n } } \langle x \rangle ^ { - 1 } \left[\left|\nabla_{x} u(s, x)\right|^{2}+\right.\right.\left.\left.(1+\mu)^{-1}\langle x\rangle^{-1}\left(|\mu u(s, x)|^{2}+\left|\partial_{t} u(s, x)\right|^{2}\right)\right] d x d s\right)^{1 / 2} \\
& \leq C(\log (2+T))^{1 / 2} \int_{0}^{T}\left\|\left(\square_{\mathbb{R}^{1+n}}+\mu^{2}\right) u(s, \cdot)\right\|_{2} d s
\end{aligned}
$$

Additionally, if $\sigma>0$ is fixed there is a constant which is independent of $\mu$ and $T$ so that

$$
\begin{gathered}
\left(\int_{0}^{T} \int_{\mathbb{R}^{n}}\langle x\rangle^{-1-\sigma}\left[\left|\nabla_{x} u(s, x)\right|^{2}+(1+\mu)^{-1}\langle x\rangle^{-1}\left(|\mu u(s, x)|^{2}+\left|\partial_{t} u(s, x)\right|^{2}\right)\right] d x d s\right)^{1 / 2} \\
\leq C \int_{0}^{T}\left\|\left(\square_{\mathbb{R}^{1+n}}+\mu^{2}\right) u(s, \cdot)\right\|_{2} d s
\end{gathered}
$$

To prove this we note that by making a dyadic decomposition of $\left\{x \in \mathbb{R}^{n}:|x| \geq 1\right\}$ and applying a scaling argument, one sees that this result follows from the following analog of Lemma 2.2 of [21].

Lemma 2.4. Let $\beta(x)$ be a fixed smooth function that is supported in $\left\{x \in \mathbb{R}^{n}:|x| \leq 1\right\}$. Assume as above that $n \geq 3$ is fixed. Then there is a constant $C$ which is independent of $\mu \geq 0$ so that

$$
\begin{array}{r}
\int_{-\infty}^{\infty}\left(\left\|\nabla_{x} \beta(\cdot)\left(e^{i t \sqrt{\mu^{2}-\Delta}} f\right)(t, \cdot)\right\|_{L^{2}\left(\mathbb{R}^{n}\right)}^{2}+(1+\mu)\left\|\beta(\cdot)\left(e^{i t \sqrt{\mu^{2}-\Delta}} f\right)(t, \cdot)\right\|_{L^{2}\left(\mathbb{R}^{n}\right)}^{2}\right) d t \\
\leq C \int_{\mathbb{R}^{n}}|\hat{f}(\xi)|^{2}\left(\mu^{2}+|\xi|^{2}\right) d \xi .
\end{array}
$$

Proof of Lemma 2.4 We just follow the proof of the special case of $\gamma=1$ of Lemma 2.2 in 21. 
We first notice that by Plancherel's theorem over $t, x$ the left side of (2.7) can be written as $(2 \pi)^{n+1}$ times

$$
\int_{\mu}^{\infty} \int\left|\int \hat{\beta}(\xi-\eta) \hat{f}(\eta) \delta\left(\tau-\sqrt{\mu^{2}+|\eta|^{2}}\right) d \eta\right|^{2}\left(1+\mu+|\xi|^{2}\right) d \xi d \tau .
$$

We next apply the Schwarz inequality over $\eta$ to bound this by

$$
\begin{aligned}
\int_{\mu}^{\infty} \int\left(1+\mu+|\xi|^{2}\right)\left[\int|\hat{\beta}(\xi-\eta)|\right. & \left.\delta\left(\tau-\sqrt{\mu^{2}+|\eta|^{2}}\right) d \eta\right] \\
\times & {\left[\int|\hat{\beta}(\xi-\eta)||\hat{f}(\eta)|^{2} \delta\left(\tau-\sqrt{\mu^{2}+|\eta|^{2}}\right) d \eta\right] d \xi d \tau . }
\end{aligned}
$$

Since $\delta\left(\tau-\sqrt{\mu^{2}+r^{2}}\right)=\frac{\tau}{\sqrt{\tau^{2}-\mu^{2}}} \delta\left(r-\sqrt{\tau^{2}-\mu^{2}}\right)$, using polar coordinates $\eta=r \omega, r>0$, $\omega \in S^{n-1}$, we see that if $\tau>\mu$ then

$$
\begin{aligned}
& \left(1+\mu+|\xi|^{2}\right) \int|\hat{\beta}(\xi-\eta)| \delta\left(\tau-\sqrt{\mu^{2}+|\eta|^{2}}\right) d \eta \\
& =c_{n}\left(1+\mu+|\xi|^{2}\right) \int_{S^{n-1}}\left|\hat{\beta}\left(\xi-\sqrt{\tau^{2}-\mu^{2}} \omega\right)\right| \tau\left(\sqrt{\tau^{2}-\mu^{2}}\right)^{n-2} d \omega \\
& \leq C_{N}\left(1+\mu+|\xi|^{2}\right)\left(1+|| \xi\left|-\sqrt{\tau^{2}-\mu^{2}}\right|\right)^{-N} \tau\left(\sqrt{\tau^{2}-\mu^{2}}\right)^{n-2}\left(1+\sqrt{\tau^{2}-\mu^{2}}\right)^{-(n-1)},
\end{aligned}
$$

for any $N$.

We conclude that there is a $C$ which is independent of $\mu$ so that the left side of (2.7) is dominated by

$$
\begin{aligned}
\left.\iint|\hat{\beta}(\xi-\eta)| \hat{f}(\eta)\right|^{2}\left(1+\mu+|\eta|^{2}\right) \sqrt{\mu^{2}+|\eta|^{2}}|\eta|^{n-2}(1+ & |\eta|)^{-(n-1)} d \eta d \xi \\
& \leq C \int|\hat{f}(\eta)|^{2}\left(\mu^{2}+|\eta|^{2}\right) d \eta
\end{aligned}
$$

using the assumption that $n \geq 3$ in the last step.

Proof of Proposition 2.3, We will only prove (2.5). Similar techniques can be used to obtain (2.6); see, e.g., [15].

First note that for $|x| \geq T$, (2.5) follows from the standard energy inequality. Next, we observe that when the norm in the left side is taken over $\{|x|<1\}$, 2.5 follows from (2.7). Indeed, by (2.7) and Duhamel's formula, we have

$$
\left\|\left|\nabla_{x} u\right|+(1+\mu)^{-1 / 2}\left(\mu|u|+\left|\partial_{t} u\right|\right)\right\|_{L^{2}([0, T] \times\{|x|<1\})} \leq C \int_{0}^{T}\left\|\left(\square_{\mathbb{R}^{1+n}}+\mu^{2}\right) u(s, \cdot)\right\|_{2} d s .
$$

Let $u_{R}(t, x)=R^{-2} u(R t, R x)$, then $\left(\left(\square+\mu^{2}\right) u\right)(R t, R x)=\left(\square+(R \mu)^{2}\right) u_{R}(t, x)$. So if we apply (2.8) with $u$ replaced by $u_{R}$ and $\mu$ replaced by $R \mu$ then we can change variables to see that the analog of (2.5) holds without the log factor if in the left the norms are taken over $[0, T] \times\{|x| \approx R\}$. If we square both sides and then sum over dyadic $R=2^{k}<T$, we get $(2.5)$. 
Remark: Although we shall not use it, the above proof yields a somewhat weaker result when $n=2$. Namely, in this case one has that the analogs of (2.5) and (2.6) hold with a constant that is independent of $\mu \geq 1$ (instead of $\mu \geq 0$ as in the $n \geq 3$ case).

To handle Neumann boundary conditions when $m=0$ in (1.1), we also require the following well known Klainerman-Sobolev estimates. See [10].

Proposition 2.5. Let $n \geq 2$, and set

$$
\{Z\}=\left\{\partial_{x}, \Omega_{j k}: 1 \leq j<k \leq n\right\} .
$$

Then if $R \geq 1$

$$
R^{(n-1) / 2} \sup _{R-1 \leq|x| \leq R}|h(t, x)| \leq C \sum_{|\alpha| \leq(n+2) / 2}\left\|Z^{\alpha} h(t, \cdot)\right\|_{L^{2}(R-2 \leq|x| \leq R+1)} .
$$

\section{Linear estimates for waveguides $\mathbb{R}_{+} \times\left(\mathbb{R}^{n} \times \Omega\right)$.}

The purpose of this section is to show that the estimates for solutions of wave and Klein-Gordon equations from the previous section carry over to the setting of waveguides if one gives up a few derivatives, depending on the dimension $d$ of $\Omega \subset \mathbb{R}^{d}$.

To prove these results we need to recall a few basic facts from the spectral theory of $\Delta_{\Omega}$ and from its elliptic regularity theory (see, e.g., 23] for proofs; see also [1]). As before, $\Delta_{\Omega}$ denotes either the Dirichlet Laplacian, in which case we impose boundary conditions

$$
\left.h\right|_{\partial \Omega}=0,
$$

or the Neumann Laplacian where we require

$$
\left.\partial_{\nu} h\right|_{\partial \Omega}=0
$$

with $\partial_{\nu}$ denoting the normal derivative.

We first recall that since we are assuming that $\Omega$ is compact with smooth boundary, the spectrum of $-\Delta_{\Omega}$ is discrete and nonnegative. We let $\lambda_{1}^{2} \leq \lambda_{2}^{2} \leq \lambda_{3}^{2} \leq \cdots$ denote the eigenvalues counted with respect to multiplicity. Then

$$
0<\lambda_{1}^{2} \leq \lambda_{2}^{2} \leq \cdots, \quad \text { for the Dirichlet Laplacian, }
$$

and

$$
0=\lambda_{1}^{2}<\lambda_{2}^{2} \leq \lambda_{3}^{2} \cdots, \quad \text { for the Neumann Laplacian. }
$$

For either of these Laplacians, we shall let $E_{j}: L^{2}(\Omega) \rightarrow L^{2}(\Omega)$ denote the projection onto the $j$ th eigenspace. Thus, if $h \in L^{2}(\Omega)$, we have that $E_{j} h$ is smooth,

$$
-\Delta_{\Omega} E_{j} h(x)=\lambda_{j}^{2} E_{j} h(x),
$$

and we have Plancherel's theorem

$$
\|h\|_{L^{2}(\Omega)}^{2}=\sum_{j=1}^{\infty}\left\|E_{j} h\right\|_{2}^{2} .
$$


Also, by the well known Weyl formula we have $\lambda_{j} \approx j^{1 / d}, j=2,3, \ldots$ Therefore, if $h \in C^{\infty}(\bar{\Omega})$,

$$
\begin{aligned}
& (1+j)^{2 / d}\left\|E_{j} h\right\|_{L^{2}(\Omega)} \leq C\left\|\left(I-\Delta_{\Omega}\right) E_{j} h\right\|_{L^{2}(\Omega)} \\
& \quad=C\left\|E_{j}\left(I-\Delta_{\Omega}\right) h\right\|_{L^{2}(\Omega)}, \quad j=1,2,3, \ldots,
\end{aligned}
$$

assuming that either (3.1) or (3.2) holds.

Having reviewed the spectral theory, let us recall the basic elliptic regularity estimate:

Lemma 3.1. Suppose that $h \in C^{\infty}(\bar{\Omega})$ and that we have the boundary conditions (3.1) or (3.2). Then for $N=2,3, \ldots$ there is a constant $C=C_{N, \Omega}$ so that

$$
\sum_{|\alpha| \leq N}\left\|\partial_{y}^{\alpha} h\right\|_{L^{2}(\Omega)} \leq C \sum_{|\alpha| \leq N-2}\left\|\partial_{y}^{\alpha} \Delta_{\Omega} h\right\|_{L^{2}(\Omega)}+C\|h\|_{L^{2}(\Omega)} .
$$

Using this result we can obtain the following

Lemma 3.2. Suppose that $u(t, x, y) \in C^{\infty}\left(\mathbb{R}_{+} \times \mathbb{R}^{n} \times \Omega\right)$ and that either $u(t, x, y)=0$ for all $y \in \partial \Omega$ or $\partial_{\nu} u(t, x, y)=0$ for all $y \in \partial \Omega$. Then if $m \geq 0$ and $N=2,4,6, \ldots$ are fixed

$$
\begin{aligned}
& \sum_{|\alpha| \leq N}\left\|\partial_{y}^{\alpha} u(t, x, \cdot)\right\|_{L^{2}(\Omega)} \\
& \quad \leq C \sum_{|\alpha| \leq N}\left\|\partial_{t, x}^{\alpha} u(t, x, \cdot)\right\|_{L^{2}(\Omega)}+C \sum_{|\alpha| \leq N-2}\left\|\partial_{t, x, y}^{\alpha}\left(\square+m^{2}\right) u(t, x, \cdot)\right\|_{L^{2}(\Omega)} .
\end{aligned}
$$

Moreover, for any $N=2,3,4, \ldots$,

$$
\begin{aligned}
& \sum_{|\alpha| \leq N}\left\|\partial_{y}^{\alpha} u(t, x, \cdot)\right\|_{L^{2}(\Omega)} \\
& \quad \leq C \sum_{\substack{|\alpha|+|\beta| \leq N \\
|\beta| \leq 1}}\left\|\partial_{t, x}^{\alpha} \partial_{y}^{\beta} u(t, x, \cdot)\right\|_{L^{2}(\Omega)}+C \sum_{|\alpha| \leq N-2}\left\|\partial_{t, x, y}^{\alpha}\left(\square+m^{2}\right) u(t, x, \cdot)\right\|_{L^{2}(\Omega)} .
\end{aligned}
$$

The proof is quite simple. Since $-\Delta_{\Omega}=\left(\square+m^{2}\right)-\partial_{t}^{2}+\Delta_{\mathbb{R}^{n}}-m^{2}$, and since $\partial_{t}$ and $\nabla_{x}$ preserve the boundary conditions, one just uses Lemma 3.1 and an induction argument.

We also require a simple lemma which shows how the spectrum of $\Delta_{\Omega}$ is relevant for our equations involving $\left(\square+m^{2}\right)$.

Proposition 3.3. Let $\Delta_{\Omega}$ denote either the Dirichlet Laplacian or the Neumann Laplacian on $\Omega$. Then, if $u \in C^{\infty}\left(\mathbb{R}_{+} \times \mathbb{R}^{n} \times \Omega\right)$ satisfies the relevant boundary condition (1.3) or (1.4), it follows that

$$
E_{j}\left(\square+m^{2}\right) u(t, x, y)=\left(\partial_{t}^{2}-\Delta_{\mathbb{R}^{n}}+m^{2}+\lambda_{j}^{2}\right) E_{j} u(t, x, y),
$$

and so

$$
\sum_{j}\left\|\left(\partial_{t}^{2}-\Delta_{\mathbb{R}^{n}}+m^{2}+\lambda_{j}^{2}\right) E_{j} u(t, x, \cdot)\right\|_{L^{2}(\Omega)}^{2}=\left\|\left(\square+m^{2}\right) u(t, x, \cdot)\right\|_{L^{2}(\Omega)}^{2} .
$$


This follows immediately from (3.5) and (3.6).

Next we need the following consequence of Sobolev's lemma for $\Omega$ and the above elliptic regularity estimates.

Proposition 3.4. Let $u$ be as above. Then if $m \geq 0$ is fixed

$$
\begin{aligned}
\left|\partial_{y}^{\beta} u(t, x, y)\right| \leq C \sum_{|\alpha| \leq|\beta|+(d+4) / 2}\left\|\partial_{t, x}^{\alpha} u(t, x, \cdot)\right\|_{L^{2}(\Omega)} & \\
& +C \sum_{|\alpha| \leq|\beta|+d / 2}\left\|\partial_{t, x, y}^{\alpha}\left(\square+m^{2}\right) u(t, x, \cdot)\right\|_{L^{2}(\Omega)} .
\end{aligned}
$$

The proof is simple. One first uses Sobolev's lemma for $\Omega$ to obtain

$$
\left|\partial_{y}^{\beta} u(t, x, y)\right| \leq C \sum_{|\alpha| \leq|\beta|+(d+2) / 2}\left\|\partial_{y}^{\alpha} u(t, x, \cdot)\right\|_{L^{2}(\Omega)} .
$$

Therefore, if we apply Lemma 3.2 (and sum over indices of length one more if $|\beta|+(d+2) / 2$ is odd), we obtain (3.8).

We can now state one of our most important estimates. We shall let

$$
\{\Gamma\}=\left\{\Gamma_{t, x}\right\} \cup\left\{\partial_{y}\right\}=\left\{\partial_{t}, \partial_{x}, \Omega_{j k}, \partial_{y}: 0 \leq j<k \leq n\right\}
$$

Proposition 3.5. Fix $B$ and suppose that $u \in C^{\infty}\left(\mathbb{R}_{+} \times \mathbb{R}^{n} \times \bar{\Omega}\right)$ satisfies $u(t, x, y)=0$, $t \leq 2 B$ and $\left(\square+m^{2}\right) u(t, x, y)=0$ if $|x|>t-B$. Suppose also that either $m \geq 0$ and $u(t, x, y)=0, y \in \partial \Omega$ or that $m>0$ and $\partial_{\nu} u(t, x, y)=0, y \in \partial \Omega$. Then, if $n=3$,

$$
\begin{array}{r}
t^{3 / 2}\left|\Gamma^{\beta} u(t, x, y)\right| \leq C \sum_{|\alpha| \leq|\beta|+5+(5 d+4) / 2} \sum_{k} \sup _{\tau \in\left[2^{k-1}, 2^{k+1}\right] \cap[2 B, t]} 2^{k}\left\|\Gamma^{\alpha}\left(\square+m^{2}\right) u(\tau, \cdot)\right\|_{2} \\
+C t^{3 / 2} \sum_{|\alpha| \leq|\beta|+(5 d+5) / 2}\left\|\Gamma^{\alpha}\left(\square+m^{2}\right) u(t, \cdot)\right\|_{2} .
\end{array}
$$

If $n=4$,

$$
\begin{aligned}
& t^{2}\left|\Gamma^{\beta} u(t, x, y)\right| \\
& \leq C \sum_{|\alpha| \leq|\beta|+7+(5 d+4) / 2} \sum_{k} \sup _{\tau \in\left[2^{k-1}, 2^{k+1}\right] \cap[2 B, t]}(1+|k|) 2^{k}\left\|\Gamma^{\alpha}\left(\square+m^{2}\right) u(\tau, \cdot)\right\|_{2} \\
& +C t^{2} \sum_{|\alpha| \leq|\beta|+(5 d+n+2) / 2}\left\|\Gamma^{\alpha}\left(\square+m^{2}\right) u(t, \cdot)\right\|_{2} .
\end{aligned}
$$

Finally, if $n \geq 5$

$$
\begin{aligned}
& t^{1+\frac{n}{4}}\left|\Gamma^{\beta} u(t, x, y)\right| \\
& \leq C \sum_{|\alpha| \leq|\beta|+n+3+(5 d+4) / 2} \sum_{k} \sup _{\tau \in\left[2^{k-1}, 2^{k+1}\right] \cap[2 B, t]} 2^{k}\left\|\Gamma^{\alpha}\left(\square+m^{2}\right) u(\tau, \cdot)\right\|_{2} \\
& +C t^{1+\frac{n}{4}} \sum_{|\alpha| \leq|\beta|+(5 d+n+2) / 2}\left\|\Gamma^{\alpha}\left(\square+m^{2}\right) u(t, \cdot)\right\|_{2} \cdot
\end{aligned}
$$

Here as above, we are assuming that $d$ is the dimension of $\Omega$. 
Proof of Proposition 3.5. To prove this, we first note that since $\left\{\Gamma_{t, x}\right\}$ commute with $\square+m^{2}$ and preserve the boundary conditions, we only have to prove the above estimates when $\Gamma^{\beta}=\partial_{y}^{\beta}$, where, as before $y$ is the $\Omega$-variable. If we apply Proposition 3.4 and orthogonality, we conclude that

$$
\begin{aligned}
& \left|\partial_{y}^{\beta} u(t, x, y)\right|^{2} \leq C \sum_{|\alpha| \leq|\beta|+(d+4) / 2}\left\|\partial_{t, x}^{\alpha} u(t, x, \cdot)\right\|_{L^{2}(\Omega)}^{2} \\
& +C \sum_{|\alpha| \leq|\beta|+d / 2}\left\|\partial_{t, x, y}^{\alpha}\left(\square+m^{2}\right) u(t, x, \cdot)\right\|_{L^{2}(\Omega)}^{2} \\
& \leq C \sum_{|\alpha| \leq|\beta|+(d+4) / 2} \sum_{j=1}^{\infty}\left\|E_{j} \partial_{t, x}^{\alpha} u(t, x, \cdot)\right\|_{L^{2}(\Omega)}^{2} \\
& +C \sum_{|\alpha| \leq|\beta|+(d+n+2) / 2}\left\|\partial_{t, x, y}^{\alpha}\left(\square+m^{2}\right) u(t, \cdot)\right\|_{L^{2}\left(\mathbb{R}^{n} \times \Omega\right)}^{2},
\end{aligned}
$$

using Sobolev's lemma for $\mathbb{R}^{n}$ in the last step.

To estimate the first term in the right, we use (3.7) to get

$$
\begin{aligned}
(1+j)^{2 / d} \sum_{|\alpha| \leq|\beta|+(d+4) / 2}\left\|E_{j} \partial_{t, x}^{\alpha} u(t, x, \cdot)\right\|_{L^{2}(\Omega)} & \\
\leq & C \sum_{|\alpha| \leq|\beta|+(d+4) / 2}\left\|E_{j}\left(I-\Delta_{\Omega}\right) \partial_{t, x}^{\alpha} u(t, x, \cdot)\right\|_{L^{2}(\Omega)} \\
\leq & C \sum_{|\alpha| \leq 2+|\beta|+(d+4) / 2}\left\|E_{j} \partial_{t, x}^{\alpha} u(t, x, \cdot)\right\|_{L^{2}(\Omega)} \\
& +C \sum_{|\alpha| \leq|\beta|+(d+4) / 2}\left\|E_{j} \partial_{t, x}^{\alpha}\left(\square+m^{2}\right) u(t, x, \cdot)\right\|_{L^{2}(\Omega)} .
\end{aligned}
$$

By induction,

$$
\begin{aligned}
\sum_{|\alpha| \leq|\beta|+(d+4) / 2}\left\|E_{j} \partial_{t, x}^{\alpha} u(t, x, \cdot)\right\|_{L^{2}(\Omega)} \leq & \frac{C}{(1+j)^{2}} \sum_{|\alpha| \leq|\beta|+(5 d+4) / 2}\left\|E_{j} \partial_{t, x}^{\alpha} u(t, x, \cdot)\right\|_{L^{2}(\Omega)} \\
& +C \sum_{|\alpha| \leq|\beta|+5 d / 2}\left\|E_{j} \partial_{t, x}^{\alpha}\left(\square+m^{2}\right) u(t, x, \cdot)\right\|_{L^{2}(\Omega)}
\end{aligned}
$$

Therefore, by (3.6) and Sobolev's lemma for $\mathbb{R}^{n}$ we conclude that

$$
\begin{aligned}
& \sum_{|\alpha| \leq|\beta|+(d+4) / 2} \sum_{j=1}^{\infty}\left\|E_{j} \partial_{t, x}^{\alpha} u(t, x, \cdot)\right\|_{L^{2}(\Omega)}^{2} \\
& \leq C \sum_{|\alpha| \leq|\beta|+(5 d+n+2) / 2}^{\infty}\left\|\partial_{t, x}^{\alpha}\left(\square+m^{2}\right) u(t, \cdot)\right\|_{L^{2}\left(\mathbb{R}^{n} \times \Omega\right)}^{2} \\
& \quad+C \sum_{j=1}^{\infty} \frac{1}{(1+j)^{4}}\left(\sum_{|\alpha| \leq|\beta|+(5 d+4) / 2}\left\|E_{j} \partial_{t, x}^{\alpha} u(t, x, \cdot)\right\|_{L^{2}(\Omega)}^{2}\right) .
\end{aligned}
$$


Thus, if we combine these two steps, we conclude that

$$
\begin{aligned}
t^{n}\left|\partial_{y}^{\beta} u(t, x, y)\right|^{2} \leq & C t^{n} \sum_{|\alpha| \leq|\beta|+(5 d+n+2) / 2}\left\|\partial_{t, x}^{\alpha}\left(\square+m^{2}\right) u(t, \cdot)\right\|_{L^{2}\left(\mathbb{R}^{n} \times \Omega\right)}^{2} \\
& +C t^{n} \sum_{j=1}^{\infty} \frac{1}{(1+j)^{4}}\left(\sum_{|\alpha| \leq|\beta|+(5 d+4) / 2}\left\|E_{j} \partial_{t, x}^{\alpha} u(t, x, \cdot)\right\|_{L^{2}(\Omega)}^{2}\right) .
\end{aligned}
$$

We can use Proposition 3.3 and Propositions 2.1 and 2.2 to estimate each of the summands in the last term since our assumptions regarding $m$ and the boundary conditions ensure that $m^{2}+\lambda_{j}^{2} \geq c$, for some constant $c>0$. When $n=3$, we get from Proposition 2.1 that there is a constant $C$ independent of $j$ such that

$$
\begin{aligned}
& t^{3 / 2} \sum_{|\alpha| \leq|\beta|+(5 d+4) / 2}\left\|E_{j} \partial_{t, x}^{\alpha} u(t, x, \cdot)\right\|_{L^{2}(\Omega)} \\
& \leq C \sum_{|\alpha| \leq|\beta|+5+(5 d+4) / 2} \sum_{k} \sup _{\substack{\tau \in\left[2^{k-1}, 2^{k+1}\right] \cap[2 B, t] \\
\times\left\|\Gamma_{t, x}^{\alpha}\left(\partial_{t}^{2}-\Delta_{\mathbb{R}^{3}}+m^{2}+\lambda_{j}^{2}\right) E_{j} u(\tau, \cdot)\right\|_{L^{2}\left(\mathbb{R}^{3} \times \Omega\right)}}} 2^{k} \sum_{|\alpha| \leq|\beta|+5+(5 d+4) / 2} \sum_{k} \sup _{\tau \in\left[2^{k-1}, 2^{k+1}\right] \cap[2 B, t]} 2^{k}\left\|\Gamma_{t, x}^{\alpha}\left(\square+m^{2}\right) u(\tau, \cdot)\right\|_{L^{2}\left(\mathbb{R}^{3} \times \Omega\right)},
\end{aligned}
$$

using Proposition 3.3 in the last step. This inequality and (3.10) yield the estimate in Proposition 3.5 for $n=3$. The estimates for $n \geq 4$ follow from a similar argument.

To prove Theorem 1.3 we need the following

Proposition 3.6. Suppose that $u \in C^{\infty}\left(\mathbb{R}_{+} \times \mathbb{R}^{n} \times \bar{\Omega}\right)$ satisifes $u(t, x, y)=0, t \leq 0$ and $\partial_{\nu} u(t, x, y)=0, y \in \partial \Omega$. Then if $n \geq 3$

$$
\begin{aligned}
& \sum_{|\alpha| \leq N}\left(\left\|\Gamma^{\alpha} \nabla_{t, x} u(T, \cdot)\right\|_{L^{2}\left(\mathbb{R}^{n} \times \Omega\right)}+(\log (2+T))^{-1 / 2}\left\|\langle x\rangle^{-1 / 2} \Gamma^{\alpha} \nabla_{x} u\right\|_{L^{2}\left([0, T] \times \mathbb{R}^{n} \times \Omega\right)}\right) \\
& \leq C \int_{0}^{T} \sum_{|\alpha| \leq N}\left\|\Gamma^{\alpha} \square u(t, \cdot)\right\|_{L^{2}\left(\mathbb{R}^{n} \times \Omega\right)} d t \\
& +C \sum_{|\alpha| \leq N-1}\left(\left\|\Gamma^{\alpha} \square u(T, \cdot)\right\|_{L^{2}\left(\mathbb{R}^{n} \times \Omega\right)}+(\log (2+T))^{-1 / 2}\left\|\langle x\rangle^{-1 / 2} \Gamma^{\alpha} \square u\right\|_{L^{2}\left([0, T] \times \mathbb{R}^{n} \times \Omega\right)}\right) .
\end{aligned}
$$

Proof of Proposition 3.6 Let us start out by showing that the second term in the left side satisfies the desired estimates. By Lemma 3.2 it suffices to prove the variant where all of the $\Gamma^{\alpha}$ are of the form $\Gamma_{t, x}^{\alpha}$. Since these vector fields preserve the boundary conditions, we see that it suffices to prove the estimate for $N=0$. But this follows from 
Proposition 2.3 and Proposition 3.3

$$
\begin{aligned}
(\log (2+T))^{-1} \| & \langle x\rangle^{-1 / 2} \nabla_{x} u \|_{L^{2}\left([0, T] \times \mathbb{R}^{n} \times \Omega\right)}^{2} \\
& =(\log (2+T))^{-1} \sum_{j=1}^{\infty}\left\|\langle x\rangle^{-1 / 2} \nabla_{x} E_{j} u\right\|_{L^{2}\left([0, T] \times \mathbb{R}^{n} \times \Omega\right)}^{2} \\
& \leq C \sum_{j=1}^{\infty}\left[\int_{0}^{T}\left\|\left(\partial_{t}^{2}-\Delta_{\mathbb{R}^{n}}+\lambda_{j}^{2}\right) E_{j} u(t, \cdot)\right\|_{L^{2}\left(\mathbb{R}^{n} \times \Omega\right)} d t\right]^{2} \\
& \leq C\left[\int_{0}^{T}\left(\sum_{j=1}^{\infty}\left\|\left(\partial_{t}^{2}-\Delta_{\mathbb{R}^{n}}+\lambda_{j}^{2}\right) E_{j} u(t, \cdot)\right\|_{L^{2}\left(\mathbb{R}^{n} \times \Omega\right)}^{2}\right)^{1 / 2} d t\right]^{2} \\
& \leq C\left[\int_{0}^{T}\|\square u(t, \cdot)\|_{L^{2}\left(\mathbb{R}^{n} \times \Omega\right)} d t\right]^{2} .
\end{aligned}
$$

This completes the proof that the second term in the left side of (3.11) satisfies the desired bounds. If one uses the energy inequality for $\mathbb{R}^{n}$, then similar arguments imply that the other term is also dominated by the right side.

One can similarly use (2.6) to show that

$$
\begin{aligned}
& \sum_{|\alpha| \leq N}\left(\left\|\Gamma^{\alpha} \nabla_{t, x} u(T, \cdot)\right\|_{L^{2}\left(\mathbb{R}^{n} \times \Omega\right)}+\left\|\langle x\rangle^{-1 / 2-\sigma} \Gamma^{\alpha} \nabla_{x} u\right\|_{L^{2}\left([0, T] \times \mathbb{R}^{n} \times \Omega\right)}\right) \\
& \leq C \int_{0}^{T} \sum_{|\alpha| \leq N}\left\|\Gamma^{\alpha} \square u(t, \cdot)\right\|_{L^{2}\left(\mathbb{R}^{n} \times \Omega\right)} d t \\
& \quad+C \sum_{|\alpha| \leq N-1}\left(\left\|\Gamma^{\alpha} \square u(T, \cdot)\right\|_{L^{2}\left(\mathbb{R}^{n} \times \Omega\right)}+\left\|\langle x\rangle^{-1 / 2-\sigma} \Gamma^{\alpha} \square u\right\|_{L^{2}\left([0, T] \times \mathbb{R}^{n} \times \Omega\right)}\right)
\end{aligned}
$$

for any $\sigma>0$.

\section{Existence theorems for Dirichlet boundary conditions.}

Proving the existence results for (1.1) with Dirichlet boundary conditions (1.3) is straightforward. To apply Proposition [3.5] as in [2], 11, one shifts the time variable by $2 B$ so that the initial condition is at $t=2 B$. Then by well known local existence theorems (see e.g. 7]) if $\varepsilon>0$ is sufficiently small and $N$ is large enough in (1.7) there is always a solution of (1.1), (1.3) on $[2 B, 2 B+1]$. To reduce to the case of zero initial data, we fix $\eta \in C^{\infty}(\mathbb{R})$ so that $\eta(t)=1, t \leq 2 B+\frac{1}{2}$ and $\eta(t)=0, t \geq 2 B+1$. Then

$$
u_{0}(t, x)=\left\{\begin{array}{l}
\eta(t) u(t, x), \quad t \leq 2 B+1 \\
0, \quad \text { otherwise }
\end{array}\right.
$$

satisfies

$$
\left(\square+m^{2}\right) u_{0}=\eta Q\left(u, u^{\prime}, u^{\prime \prime}\right)+[\square, \eta] u
$$


with Dirichlet boundary conditions. Therefore, $\left(\square+m^{2}\right) u=Q\left(u, u^{\prime}, u^{\prime \prime}\right)$ for $2 B<t<T$ and $(x, y) \in \mathbb{R}^{n} \times \Omega$ if and only if $w=u-u_{0}$ solves

$$
\left\{\begin{array}{l}
\left(\square+m^{2}\right) w=(1-\eta) Q\left(u_{0}+w,\left(u_{0}+w\right)^{\prime},\left(u_{0}+w\right)^{\prime \prime}\right)-[\square, \eta]\left(u_{0}+w\right) \\
w(t, x, y)=0, \quad y \in \partial \Omega \\
w(t, x, y)=0, \quad t \leq 2 B .
\end{array}\right.
$$

Since the local existence results imply that

$$
\sup _{t} \sum_{|\alpha| \leq N}\left\|\Gamma^{\alpha} u_{0}(t, \cdot)\right\|_{L^{2}\left(\mathbb{R}^{n} \times \Omega\right)} \leq C \varepsilon,
$$

one can argue as in Hörmander [2] $\S 7.4$ that if $N$ is large enough and if $\varepsilon$ is small enough then there is a global solution of (4.1) if $n \geq 3$. One uses Proposition [3.5] and energy estimates. The latter say that if $\gamma^{j k} \in C^{\infty}$,

$$
\sum_{j, k=0}^{n+d}\left|\gamma^{j k}\right| \leq 1 / 2
$$

and if

$$
\left\{\begin{array}{l}
\left(\square+m^{2}\right) w+\sum_{j, k=0}^{n+d} \gamma^{j k}(t, x, y) \partial_{j} \partial_{k} w=F, \quad 2 B \leq t \leq T \\
w(t, x, y)=0, \quad y \in \partial \Omega \\
w(t, x, y)=0, \quad t \leq 2 B
\end{array}\right.
$$

then if $w$ vanishes for large $|x|$ we have

$$
\begin{aligned}
& \left\|\nabla_{t, x, y} w(t, \cdot)\right\|_{2}+m\|w(t, \cdot)\|_{2} \\
& \quad \leq 2 \exp \left(\int_{0}^{t} 2 \sum_{i, j, k=0}^{n+d}\left\|\partial_{i} \gamma^{j k}(s, \cdot)\right\|_{\infty} d s\right) \int_{0}^{t}\|F(s, \cdot)\|_{2} d s .
\end{aligned}
$$

Here, as before, $x_{0}=t$, while $x_{n+j}=y_{j}, 1 \leq j \leq d$. Since $w$ has Dirichlet boundary conditions the proof of Lemma 7.4.1 in [2] shows that (4.3) holds.

In order to use the well-known arguments of Klainerman [1] to show global existence, we will require a version of (4.3) where $w$ is replaced by $\Gamma^{\alpha} w$. We begin with the case $\Gamma=\partial_{y}$. Here, since $\nabla_{t, x}$ preserves the boundary condition, it follows from Lemma 3.2 that if $|\alpha|=N$,

$$
\begin{aligned}
& \left\|\partial_{y}^{\alpha} \nabla_{t, x, y} w(t, \cdot)\right\|_{2}+m\left\|\partial_{y}^{\alpha} w(t, \cdot)\right\|_{2} \\
& \leq C \sum_{\substack{|\beta|+|\gamma| \leq N+1 \\
|\gamma| \leq 1}}\left\|\partial_{t, x}^{\beta} \partial_{y}^{\gamma} w(t, \cdot)\right\|_{2}+C \sum_{|\beta| \leq N-1}\left\|\partial_{t, x, y}^{\beta}\left(\square+m^{2}\right) w(t, \cdot)\right\|_{2} .
\end{aligned}
$$

Thus, since the vectors fields $\Gamma_{t, x}$ preserve the boundary conditions and

$$
\begin{aligned}
\left(\square+m^{2}\right) \Gamma_{t, x}^{\alpha} w+\sum_{j, k=0}^{n+d} \gamma^{j k} \partial_{j} \partial_{k} \Gamma_{t, x}^{\alpha} w & \\
& =\Gamma_{t, x}^{\alpha} F+\sum_{j, k=0}^{n+d}\left[\gamma^{j k}, \Gamma_{t, x}^{\alpha}\right] \partial_{j} \partial_{k} w+\sum_{j, k=0}^{n+d} \gamma^{j k}\left[\partial_{j} \partial_{k}, \Gamma_{t, x}^{\alpha}\right] w,
\end{aligned}
$$


it follows easily from the proof of Lemma 7.4.1 in [2] and (4.4) that for $N=0,1,2, \ldots$,

$$
\begin{aligned}
& \sum_{|\alpha| \leq N}\left\|\Gamma^{\alpha} \nabla_{t, x, y} w(t, \cdot)\right\|_{2}+m \sum_{|\alpha| \leq N}\left\|\Gamma^{\alpha} w(t, \cdot)\right\|_{2} \\
& \leq C \exp \left(\int_{0}^{t} 2 \sum_{i, j, k=0}^{n+d}\left\|\partial_{i} \gamma^{j k}(s, \cdot)\right\|_{\infty} d s\right) \sum_{|\alpha| \leq N} \int_{0}^{t}\left\|\Gamma_{t, x}^{\alpha} F(s, \cdot)\right\|_{2} d s \\
&+C \exp \left(\int_{0}^{t} 2 \sum_{i, j, k=0}^{n+d}\left\|\partial_{i} \gamma^{j k}(s, \cdot)\right\|_{\infty} d s\right) \sum_{|\alpha| \leq N} \sum_{j, k=0}^{n+d} \int_{0}^{t}\left\|\left[\gamma^{j k}, \Gamma_{t, x}^{\alpha}\right] \partial_{j} \partial_{k} w(s, \cdot)\right\|_{2} d s \\
&+C \sum_{|\beta| \leq N-1}\left\|\partial_{t, x, y}^{\beta}\left(\square+m^{2}\right) w(t, \cdot)\right\|_{2} .
\end{aligned}
$$

Standard arguments show that (4.5) and Proposition 3.5]imply the existence results for the nonlinear Dirichlet-wave equations (1.1) when $m>0$. Since Proposition 3.5 remains valid in the Dirichlet case when $m=0$, one also gets the existence results for $m=0$. This requires that $\|w(t, \cdot)\|_{2}$ is dominated by the right side of (4.3) in the Dirichlet case due to the fact that Poincaré's lemma gives $\|w(t, \cdot)\|_{2} \leq C\left\|\nabla_{y} w(t, \cdot)\right\|_{2}$.

\section{Existence theorems for Neumann boundary conditions.}

The proof of Theorem 1.2 follows from the same arguments that were just used for the proof of Theorem 1.1. Since one has Neumann boundary conditions, though, one has to make an extra assumption in order for (4.3) to hold for solutions of

$$
\left\{\begin{array}{l}
\square w+\sum_{j, k=0}^{n+d} \gamma^{j k}(t, x, y) \partial_{j} \partial_{k} w+m^{2} w=F, \quad 2 B \leq t \leq T \\
\partial_{\nu} w(t, x, y)=0, \quad y \in \partial \Omega \\
w(t, x, y)=0, \quad t \leq 2 B .
\end{array}\right.
$$

The necessary assumption is that

$$
\begin{aligned}
\sum_{0 \leq j, k \leq n+d} \gamma^{j k}(t, x, y) \xi_{j} \theta_{k}=0, \text { if } y & \in \partial \Omega, \\
\theta & =\left(0, \ldots, 0, \nu_{1}(y), \ldots \nu_{d}(y)\right) \text {, and } \xi \cdot \nu(y)=0 .
\end{aligned}
$$

Under this assumption, the proof of Lemma 7.4.1 in 2] shows that 4.3] is valid when $w$ is a solution of (5.1). The assumption (1.8) for the nonlinear terms implies that if $\partial_{\nu} u(t, x, y)=0, y \in \partial \Omega$ then

$$
\gamma^{j k}(t, x, y)=\sum_{l=0}^{n+d} A_{l}^{j k} \partial_{l} u+u A^{j k}
$$

must satisfy (5.2).

Since we have just seen that we can apply (4.3), (4.5) under the assumption (1.8), we conclude that standard arguments give Theorem 1.2 i.e., the existence results for our nonlinear Klein-Gordon equations with Neumann boundary conditions.

For Theorem 1.3 which concerns existence for semilinear wave equations with Neumann boundary conditions, one just needs to use the special case of (4.3), (4.5) where all 
of the $\gamma^{j k}$ vanish identically, i.e., the standard energy estimate for constant coefficients and Neumann conditions. By using this and Proposition 3.6 one obtains Theorem 1.3 using arguments from [8]. The remainder of this section will be dedicated to sketching this proof.

Proof of Theorem 1.3: Using the reduction outline at the beginning of the preceding section, it suffices to show that the solution $w$ to

$$
\left\{\begin{array}{l}
\square w=(1-\eta) Q\left(\nabla_{x}\left(u_{0}+w\right)\right)-[\square, \eta]\left(u_{0}+w\right) \\
\partial_{\nu} w(t, x, y)=0, \quad y \in \partial \Omega \\
w(t, x, y)=0, \quad t \leq 2 B
\end{array}\right.
$$

exists for $t \in\left[0, T_{\varepsilon}\right)$ when $n=3$ and exists globally for $n \geq 4$. We solve this equation using iteration. To do so, we then let $w_{0} \equiv 0$ and set $w_{k}$ to be the solution of

$$
\left\{\begin{array}{l}
\square w_{k}=(1-\eta) Q\left(\nabla_{x}\left(u_{0}+w_{k-1}\right)\right)-[\square, \eta]\left(u_{0}+w_{k}\right) \\
\partial_{\nu} w_{k}(t, x, y)=0, \quad y \in \partial \Omega \\
w_{k}(t, x, y)=0, \quad t \leq 2 B
\end{array}\right.
$$

for $k=1,2,3, \ldots$

We will focus on providing a sketch of the proof of Theorem 1.3 for the $n=3$ case using arguments of 8 . Similar iteration techniques can be used to prove the $n \geq 4$; see, e.g., [15].

We let

$$
\begin{aligned}
M_{k}(T)=\sup _{0 \leq t \leq T} \sum_{|\alpha| \leq 10}\left(\left\|\Gamma^{\alpha} \nabla_{t, x} w_{k}(t, \cdot)\right\|_{L^{2}\left(\mathbb{R}^{3} \times \Omega\right)}\right. & \\
& \left.+(\ln (2+t))^{-1 / 2}\left\|\langle x\rangle^{-1 / 2} \Gamma^{\alpha} \nabla_{x} w_{k}\right\|_{L^{2}\left([0, t] \times \mathbb{R}^{3} \times \Omega\right)}\right) .
\end{aligned}
$$

Our first goal is to inductively prove that for $\varepsilon$ sufficiently small we have

$$
M_{k}(T) \leq 4 C_{0} \varepsilon
$$

for a uniform constant $C_{0}$ greater than the constant occuring in (4.2), $T \in\left[2 B, T_{\varepsilon}\right.$ ), and $k=1,2,3, \ldots$. When $k=1$, (5.6) follows from (1.7), well-known local estimates, (3.11), and Gronwall's inequality.

Let us prove (5.6) inductively. Thus, we assume that this bound holds for $k-1$. By (3.11) and 4.2),

$$
\begin{aligned}
& M_{k}\left(T_{\varepsilon}\right) \leq C \sum_{|\alpha| \leq 10} \int_{0}^{T_{\varepsilon}}\left\|\Gamma^{\alpha} Q\left(\nabla_{x}\left(u_{0}+w_{k-1}\right)\right)(t, \cdot)\right\|_{L^{2}\left(\mathbb{R}^{3} \times \Omega\right)} d t \\
& +C \sum_{|\alpha| \leq 9}\left(\left\|\Gamma^{\alpha} Q\left(\nabla_{x}\left(u_{0}+w_{k-1}\right)\left(T_{\varepsilon}, \cdot\right)\right)\right\|_{L^{2}\left(\mathbb{R}^{3} \times \Omega\right)}\right. \\
& \left.+\left(\log \left(2+T_{\varepsilon}\right)\right)^{-1 / 2}\left\|\langle x\rangle^{-1 / 2} \Gamma^{\alpha} Q\left(\nabla_{x}\left(u_{0}+w_{k-1}\right)\right)\right\|_{L^{2}\left(\left[0, T_{\varepsilon}\right] \times \mathbb{R}^{3} \times \Omega\right)}\right) \\
& 2 C_{0} \varepsilon+C \int_{2 B+(1 / 2)}^{2 B+1}\left\|\Gamma^{\alpha} \partial_{t} w_{k}(t, \cdot)\right\|_{L^{2}\left(\mathbb{R}^{3} \times \Omega\right)} d t .
\end{aligned}
$$


Since $Q$ is quadratic, we can apply (2.9) and the standard Sobolev lemma to see that

$$
\begin{aligned}
& \sum_{|\alpha| \leq 10} \| \Gamma^{\alpha} Q\left(\nabla_{x}\left(u_{0}+w_{k-1}\right)(t, \cdot) \|_{L^{2}\left(\left\{2^{j} \leq|x| \leq 2^{j+1}\right\} \times \Omega\right)}\right. \\
& \leq C \sum_{|\alpha| \leq 10}\left\|\langle x\rangle^{-1 / 2} \Gamma^{\alpha} \nabla_{x} u_{0}(t, \cdot)\right\|_{L^{2}\left(\left\{2^{j} \leq|x| \leq 2^{j+1}\right\} \times \Omega\right)}^{2} \\
& +C\left(\sum_{|\alpha| \leq 10}\left\|\Gamma^{\alpha} \nabla_{x} u_{0}(t, \cdot)\right\|_{L^{2}\left(\mathbb{R}^{3} \times \Omega\right)}\right)\left(\sum_{|\alpha| \leq 10}\left\|\Gamma^{\alpha} \nabla_{x} w_{k-1}(t, \cdot)\right\|_{L^{2}\left(\left\{2^{j} \leq|x| \leq 2^{j+1}\right\} \times \Omega\right)}\right) \\
& +C\left(\sum_{|\alpha| \leq 10}\left\|\Gamma^{\alpha} \nabla_{x} u_{0}(t, \cdot)\right\|_{L^{2}\left(\left\{2^{j} \leq|x| \leq 2^{j+1}\right\} \times \Omega\right)}\right)\left(\sum_{|\alpha| \leq 10}\left\|\Gamma^{\alpha} \nabla_{x} w_{k-1}(t, \cdot)\right\|_{L^{2}\left(\mathbb{R}^{3} \times \Omega\right)}\right) \\
& +C \sum_{|\alpha| \leq 10}\left\|\langle x\rangle^{-1 / 2} \Gamma^{\alpha} \nabla_{x} w_{k-1}(t, \cdot)\right\|_{L^{2}\left(\left\{2^{j-1} \leq|x| \leq 2^{j+2}\right\} \times \Omega\right)}^{2}
\end{aligned}
$$

Thus, since $u_{0}$ vanishes for $t \geq 2 B+1$, summing over $j$ and applying (4.2) gives

$$
\int_{0}^{T_{\varepsilon}} \| \Gamma^{\alpha} Q\left(\nabla_{x}\left(u_{0}+w_{k-1}\right)(t, \cdot) \|_{L^{2}\left(\mathbb{R}^{3} \times \Omega\right)} d t \leq C\left[\varepsilon+\left(\ln \left(2+T_{\varepsilon}\right)\right)^{1 / 2} M_{k-1}\left(T_{\varepsilon}\right)\right]^{2} .\right.
$$

Using similar techniques, one can show that the second and third terms satisfy better bounds and are controlled by $C\left(\varepsilon+M_{k-1}\left(T_{\varepsilon}\right)\right)^{2}$. Hence, by Gronwall's inequality and the inductive hypothesis (5.6), we have

$$
M_{k}\left(T_{\varepsilon}\right) \leq 2 C_{0} \varepsilon+3 C\left[\varepsilon+\left(\ln \left(2+T_{\varepsilon}\right)\right)^{1 / 2} 4 C_{0} \varepsilon\right]^{2} .
$$

Thus, one can choose an appropriate $c$ in (1.10) so that this is bounded by $4 C_{0} \varepsilon$ as desired.

To show that the sequence $w_{k}$ converges to a solution, we estimate

$$
\begin{aligned}
A_{k}(T)=\sup _{0 \leq t \leq T} \sum_{|\alpha| \leq 10}\left(\left\|\Gamma^{\alpha} \nabla_{t, x}\left(w_{k}-w_{k-1}\right)(t, \cdot)\right\|_{L^{2}\left(\mathbb{R}^{3} \times \Omega\right)}\right. \\
\left.\quad+(\ln (2+t))^{-1 / 2}\left\|\langle x\rangle^{-1 / 2} \Gamma^{\alpha} \nabla_{x}\left(w_{k}-w_{k-1}\right)\right\|_{L^{2}\left([0, t] \times \mathbb{R}^{3} \times \Omega\right)}\right) .
\end{aligned}
$$

If one shows that $A_{k}$ satisfies

$$
A_{k}\left(T_{\varepsilon}\right) \leq \frac{1}{2} A_{k-1}\left(T_{\varepsilon}\right), \quad k=1,2,3, \ldots,
$$

the proof of Theorem 1.3 would be complete. Indeed this is the case. Since $Q$ is quadratic, we can repeat the previous arguments to see that

$$
A_{k}\left(T_{\varepsilon}\right) \leq C\left(2 C_{0} \varepsilon+\left(\ln \left(2+T_{\varepsilon}\right)\right)^{1 / 2}\left(M_{k-1}\left(T_{\varepsilon}\right)+M_{k-2}\left(T_{\varepsilon}\right)\right)\right)\left(\ln \left(2+T_{\varepsilon}\right)\right)^{1 / 2} A_{k-1}\left(T_{\varepsilon}\right) .
$$

By our choise of $T_{\varepsilon}$, we see that this leads to (5.9) for $\varepsilon$ sufficiently small, which completes the proof of the $n=3$ case.

In order to show global existence for $n \geq 4$, we instead use (3.12) to bound

$$
M_{k}(T)=\sup _{0 \leq t \leq T} \sum_{|\alpha| \leq n+10}\left(\left\|\Gamma^{\alpha} \nabla_{t, x} w_{k}(t, \cdot)\right\|_{2}+\left\|\langle x\rangle^{-(n-1) / 4} \Gamma^{\alpha} \nabla_{x} w_{k}\right\|_{L^{2}\left([0, t] \times \mathbb{R}^{n} \times \Omega\right)}\right)
$$


for any $T \in[2 B, \infty)$. Similarly, we show that

$$
\begin{aligned}
A_{k}(T)=\sup _{0 \leq t \leq T} \sum_{|\alpha| \leq n+10}\left(\left\|\Gamma^{\alpha} \nabla_{t, x}\left(w_{k}-w_{k-1}\right)(t, \cdot)\right\|_{L^{2}\left(\mathbb{R}^{n} \times \Omega\right)}\right. \\
\left.+\left\|\langle x\rangle^{-(n-1) / 4} \Gamma^{\alpha} \nabla_{x}\left(w_{k}-w_{k-1}\right)\right\|_{L^{2}\left([0, t] \times \mathbb{R}^{n} \times \Omega\right)}\right)
\end{aligned}
$$

is a Cauchy sequence, which would complete the proof.

\section{REFERENCES}

[1] D. Gilbarg and N. Trudinger: Elliptic partial differential equations of second order, Springer, Second Ed., Third Printing, 1998.

[2] L. Hörmander: Lectures on nonlinear hyperbolic equations, Springer-Verlag, Berlin, 1997.

[3] M. Ikawa: Decay of solutions of the wave equation in the exterior of two convex bodies. Osaka J. Math. 19 (1982), 459-509.

[4] M. Ikawa: Decay of solutions of the wave equation in the exterior of several convex bodies. Ann. Inst. Fourier (Grenoble) 38 (1998), 113-146.

[5] F. John: Blow-up for quasi-linear wave equations in three space dimensions. Comm. Pure Appl. Math. 34 (1981), 29-51.

[6] F. John: Nonlinear wave equations, formation of singularities. Amer. Math. Soc., Providence, 1990.

[7] M. Keel, H. Smith, and C. D. Sogge: Global existence for a quasilinear wave equation outside of star-shaped domains. J. Funct. Anal. 189 (2002), 155-226.

[8] M. Keel, H. Smith, and C. D. Sogge: Almost global existence for some semilinear wave equations. J. D'Analyse 87 (2002), 265-279.

[9] M. Keel, H. Smith, and C. D. Sogge: Almost global existence for quasilinear wave equations in three space dimensions. J. Amer. Math. Soc. 17 (2004), 109-153.

[10] S. Klainerman: Uniform decay estimates and the Lorentz invariance of the classical wave equation. Comm. Pure Appl. Math. 38 (1985), 321-332.

[11] S. Klainerman: Global existence of small amplitude solutions to nonlinear Klein-Gordon equations in four space-time dimensions. Comm. Pure Appl. Math. 38 (1985), 631-641.

[12] P. D. Lax, C. S. Morawetz, and R. S. Phillips: Exponential decay of solutions of the wave equation in the exterior of a star-shaped obstacle. Comm. Pure Appl. Math. 16 (1963), 477-486.

[13] P. H. Lesky and R. Racke: Nonlinear wave equations in infinite waveguides. Comm. Partial Differential Equations 28 (2003), 1265-1301.

[14] B. Marshall, W. Strauss, and S. Wainger: $L^{p}-L^{q}$ estimates for the Klein-Gordon equation. J. Math. Pures Appl. 59 (1980), 417-440.

[15] J. Metcalfe: Global existence for semilinear wave equations exterior to nontrapping obstacles. Houston J. Math. 30 (2004), 259-281.

[16] J. Metcalfe and C. D. Sogge: Hyperbolic trapped rays and global existence of quasilinear wave equations. Invent. Math. 159 (2005), 75-117.

[17] C. S. Morawetz, J. Ralston, and W. Strauss: Decay of solutions of the wave equation outside nontrapping obstacles. Comm. Pure Appl. Math. 30 (1977), 447-508.

[18] J. Shatah: Normal forms and quadratic nonlinear Klein-Gordon equations. Comm. Pure Appl. Math. 38 (1985), 685-696.

[19] Y. Shibata and Y. Tsutsumi: On a global existence theorem of small amplitude solutions for nonlinear wave equations in an exterior domain. Math. Z. 191 (1986), 165-199.

[20] T. Sideris: Nonresonance and global existence of prestressed nonlinear elastic waves. Ann. of Math. 151 (2000), 849-874.

[21] H. Smith and C. D. Sogge: Global Strichartz estimates for nontrapping perturbations of the Laplacian. Comm. Partial Differential Equations 25 (2000), 2171-2183.

[22] C. D. Sogge: Lectures on nonlinear wave equations, International Press, Cambridge, MA, 1995.

[23] M. Taylor: Partial Differential Equations I, Springer-Verlag, New York, 1996. 
School of Mathematics, Georgia Institute of Technology, Atlanta, GA 30332-0160

Department of Mathematics, Johns Hopkins University, Baltimore, MD 21218

Department of Mathematics, Johns Hopkins University, Baltimore, MD 21218 